\title{
Erratum to: Coffee or tea consumption and the risk of rheumatoid arthritis: a meta-analysis
}

\author{
Young Ho Lee • Sang-Cheol Bae • Gwan Gyu Song
}

Published online: 10 January 2015

(C) International League of Associations for Rheumatology (ILAR) 2015

\section{Erratum to: Clin Rheumatol (2014) 33:1575-1583 DOI 10.1007/s10067-014-2631-1}

We found errors in the statistical analysis of the meta-analysis on combining cohort studies and case-control studies. We corrected an error by placing a 14.2 with a 1.2 in the calculations of relative risk (RR) of Karlson et al. study. Thus, the magnitude of effect for all RA was 2.426 in the original text, but is 1.217 in the revised text. The magnitude for analysis by study design for the cohort studies was 4.148 , and now is 1.309. However, the corrected results did not change the statistical significance of the previous results and conclusion of the meta-analysis. We added a meta-analysis figure for decaffeinated coffee and one for caffeinated coffee to the erratum (Fig. 3). We added a revised summary paragraph for the erratum.

A revised summary: Meta-analysis of the cohort studies revealed a trend of an association between total coffee intake

The online version of the original article can be found at http://dx.doi.org/ 10.1007/s10067-014-2631-1.

\section{Y. H. Lee $(\bowtie) \cdot$ G. G. Song}

Division of Rheumatology, Department of Internal Medicine, Korea University Anam Hospital, Korea University College of Medicine, 126-1, Anam-dong 5-ga, Seongbuk-gu, Seoul 136-705, South Korea e-mail: lyhcgh@korea.ac.kr

\section{S.-C. Bae}

The Hospital for Rheumatic Diseases, Hanyang University Medical Center, Seoul, South Korea and RA incidence (RR of the highest vs. the lowest group $=1.309,95 \%$ confidence interval $[\mathrm{CI}]=0.967-$ $1.771, p=0.085$ ). Meta-analysis of case-control studies showed a significant association between total coffee intake and RA incidence $(\mathrm{RR}=1.201,95 \% \mathrm{CI}=$ $1.058-1.361, p=0.005)$. There were differences in the reference groups (all categories of coffee) between the case-control meta-analysis that showed a significant association and the cohort studies where meta-analysis results were non-significant. In addition, the highest category of coffee intake varied between Heliovaara et al. cohort study from Finland where the highest category included drinking up to 13 cups per day, compared to US studies where it was very unusual to have $>4$ cups coffee intake per day. Combining the data of the cohort and case-control studies showed a significant association between total coffee intake and RA incidence $(\mathrm{RR}=1.217,95 \% \mathrm{CI}=1.083-1.368$, $p=0.001)$. Meta-analysis stratified by seropositivity indicated a significant association between coffee consumption and seropositive RA risk ( $\mathrm{RR}=1.309,95 \%$ $\mathrm{CI}=1.142-1.499, p=1.1 \times 10-5)$, but not seronegative $\mathrm{RA}$ risk $(\mathrm{RR}=1.097,95 \% \mathrm{CI}=0.886-1.357, p=0.396)$. There was no significant association between decaffeinated coffee consumption and RA incidence $(R R=1.709$, $95 \%$ CI 0.786-3.715), or between caffeinated coffee consumption and RA incidence $(\mathrm{RR}=1.055,95 \% \mathrm{CI}$ $0.782-1.421)$

We apologize for this mistake. The corrected Tables 2 and 3 and Figs. 1, 2 and 3 are presented as follows: 
Table 2. Meta-analysis of studies on coffee or tea intake and the incidence of rheumatoid arthritis

\begin{tabular}{|c|c|c|c|c|c|c|c|c|c|c|}
\hline \multirow[t]{2}{*}{ Coffee intake (cups/day) } & \multirow[t]{2}{*}{ No. of Studies } & \multirow[t]{2}{*}{ Study design } & \multicolumn{2}{|c|}{ Person-years and/or subjects } & \multicolumn{3}{|c|}{ Test of association } & \multicolumn{3}{|c|}{ Test of heterogeneity } \\
\hline & & & Highest (or case) & Lowest (or control) & $\mathrm{RR}$ & $95 \% \mathrm{CI}$ & $\mathrm{p}$ value & Model & $\mathrm{p}$ value & $I^{2}$ \\
\hline \multirow[t]{3}{*}{ Total coffee } & 5 & Both & 331,573 & 237,094 & 1.217 & $1.083-1.368$ & 0.001 & $\mathrm{~F}$ & 0.791 & 0 \\
\hline & 3 & Cohort & 330,970 & 236,149 & 1.309 & $0.967-1.771$ & 0.085 & $\mathrm{~F}$ & 0.502 & 0 \\
\hline & 2 & Case-control & 603 & 945 & 1.201 & $1.058-1.364$ & 0.005 & $\mathrm{~F}$ & 0.810 & 0 \\
\hline Caffeinated & 2 & Cohort & 285,251 & 340,340 & 1.055 & $0.782-1.421$ & 0.727 & $\mathrm{~F}$ & 0.715 & 0 \\
\hline Decaffeinated & 2 & Cohort & 46,114 & 522,601 & 1.709 & $0.786-3.715$ & 0.176 & $\mathrm{R}$ & 0.062 & 71.2 \\
\hline \multirow[t]{3}{*}{ Caffeine, $\mathrm{mg}$, or ml/day } & 3 & Both & 397,779 & 371,218 & 1.074 & $0.864-1.334$ & 0.521 & $\mathrm{~F}$ & 0.563 & 0 \\
\hline & 2 & Cohort & 397,691 & 371,042 & 0.980 & $0.744-1.291$ & 0.885 & $\mathrm{~F}$ & 0.836 & 0 \\
\hline & 1 & Case-control & 88 & 176 & 1.245 & $0.876-1.769$ & 0.221 & NA & NA & NA \\
\hline \multirow[t]{3}{*}{ Tea } & 3 & Both & 88,958 & 437,269 & 0.983 & $0.830-1.164$ & 0.839 & $\mathrm{~F}$ & 0.116 & 53 \\
\hline & 2 & Cohort & 88,870 & 437,093 & 0.679 & $0.224-2.056$ & 0.493 & $\mathrm{R}$ & 0.043 & 75.5 \\
\hline & 1 & Case-control & 88 & 176 & 1.000 & $0.583-1.716$ & 1.000 & NA & NA & NA \\
\hline
\end{tabular}

Both: Cohort and case-control studies; $C I$ confidence interval; $R R$ Relative risk; $F$ Fixed effects model; $R$ Random effects model; $N A$ Not available.

Table 3. Meta-analysis of studies on coffee intake and the incidence of seropositive or seronegative RA

\begin{tabular}{|c|c|c|c|c|c|c|c|c|c|c|}
\hline \multirow[t]{2}{*}{ Population } & \multirow[t]{2}{*}{ No. of Studies } & \multirow[t]{2}{*}{ Study design } & \multicolumn{2}{|c|}{ Person-years and/or subjects } & \multicolumn{3}{|c|}{ Test of association } & \multicolumn{3}{|c|}{ Test of heterogeneity } \\
\hline & & & $\begin{array}{l}\text { Highest } \\
\text { (or case) }\end{array}$ & $\begin{array}{l}\text { Lowest } \\
\text { (or control) }\end{array}$ & $\mathrm{RR}$ & $95 \% \mathrm{CI}$ & $\mathrm{p}$ value & Model & $\mathrm{p}$ value & $I^{2}$ \\
\hline \multirow[t]{3}{*}{ Seropositive RA } & 3 & Both & 193,796 & 204,555 & 1.309 & $1.142-1.499$ & $1.1 \times 10^{-5}$ & $\mathrm{~F}$ & 0.485 & 0 \\
\hline & 2 & Cohort & 193,487 & 203,788 & 1.580 & $0.981-2.546$ & 0.060 & $\mathrm{~F}$ & 0.374 & 0 \\
\hline & 1 & Case-control & 309 & 767 & 1.287 & $1.116-1.483$ & 0.001 & NA & NA & NA \\
\hline \multirow[t]{3}{*}{ Seronegative RA } & 2 & Both & 14,010 & 1,763 & 1.097 & $0.886-1.357$ & 0.396 & $\mathrm{~F}$ & 0.828 & 0 \\
\hline & 1 & Cohort & 13,874 & 996 & 1.390 & $0.161-11.99$ & 0.765 & NA & NA & $\mathrm{N}$ \\
\hline & 1 & Case-control & 136 & 767 & 1.094 & $0.883-1.355$ & 0.411 & NA & NA & NA \\
\hline
\end{tabular}

Both: Cohort and case-control studies; $R A$ rheumatoid arthritis; $C I$ confidence interval; $R R$ Relative risk; $F$ Fixed effects model; $R$ Random effects model; $N A$ Not available.

A

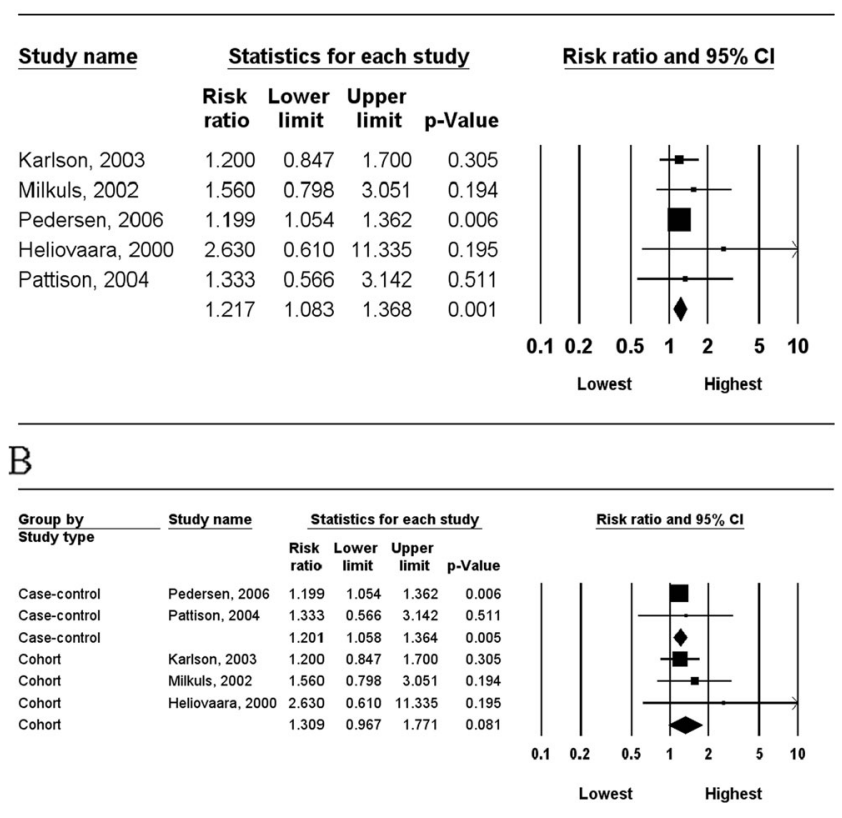

Fig. 1 Meta-analysis of the association between coffee consumption and rheumatoid arthritis risk for the highest versus lowest groups of coffee intake in the overall group (A) and each study design group (B)

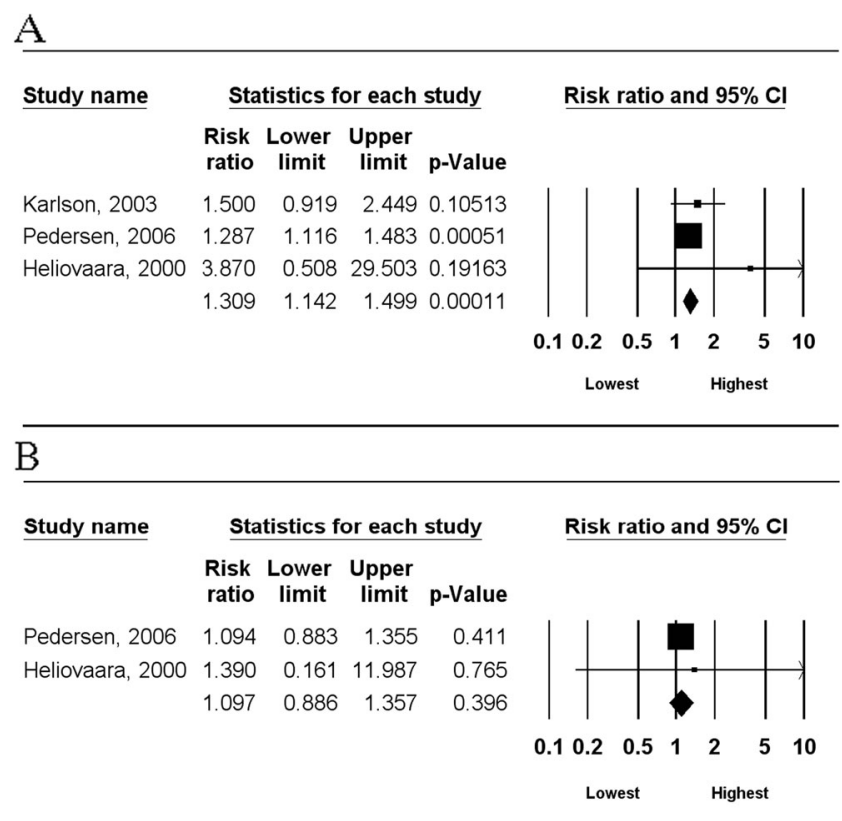

Fig. 2 Meta-analysis of the association between coffee consumption and seropositive (A) or seronegative (B) rheumatoid arthritis risk for the highest versus lowest groups of coffee intake 
A

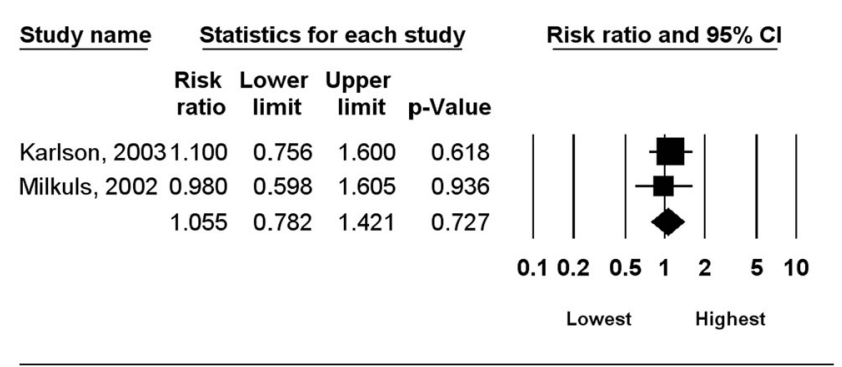

\begin{tabular}{|c|c|c|c|c|c|}
\hline \multicolumn{6}{|l|}{ B } \\
\hline \multirow[t]{2}{*}{ Study name } & \multicolumn{4}{|c|}{ Statistics for each study } & Risk ratio and $95 \% \mathrm{Cl}$ \\
\hline & $\begin{array}{l}\text { Risk } \\
\text { ratio }\end{array}$ & $\begin{array}{l}\text { Lower } \\
\text { limit }\end{array}$ & $\begin{array}{c}\text { Upper } \\
\text { limit }\end{array}$ & p-Value & \\
\hline Karlson, 2003 & 1.100 & 0.550 & 2.200 & 0.788 & \\
\hline \multirow[t]{4}{*}{ Milkuls, 2002} & 2.440 & 1.525 & 3.903 & 0.000 & \\
\hline & 1.709 & 0.786 & 3.715 & 0.176 & \\
\hline & & & & & $\begin{array}{lllll}0.1 & 0.2 & 0.5 & 1 & 2\end{array}$ \\
\hline & & & & & Highest \\
\hline
\end{tabular}

Fig. 3 Meta-analysis of the association between caffeinated (A) or decaffeinated (B) coffee and rheumatoid arthritis risk for the highest versus lowest groups of coffee intake 